\title{
Abnormal microtubule dynamics disrupt nucleocytoplasmic transport in tau- mediated frontotemporal dementia
}

Paonessa, F.1,2, Evans, L.1,2, Solanki, R ${ }^{1,2}$., Larrieu, D. ${ }^{1,4}$, Wray, S. ${ }^{3}$, Hardy, J. ${ }^{3}$, Jackson, S.P. ${ }^{1}$ and F.J. Livesey ${ }^{1,2^{*}}$

${ }^{1}$ Gurdon Institute, University of Cambridge, Tennis Court Road, Cambridge, CB2 1QN

${ }^{2}$ Alzheimer's Research UK Stem Cell Research Centre, University of Cambridge, CB2 $1 Q N$

${ }^{3}$ Department of Molecular Neuroscience, UCL Institute of Neurology, Queen Square, London WC1N 3BG, UK

${ }^{4}$ Current address: Cambridge Institute for Medical Research, University of Cambridge, Hills Road CB2 OXY, Cambridge

*Author for correspondence: rick@gurdon.cam.ac.uk 


\section{SUMMARY}

The neuronal microtubule-associated protein tau $(M A P T)$ is central to the pathogenesis of many dementias, including Alzheimer's disease. Autosomal dominant mutations in MAPT cause inherited frontotemporal dementia (FTD), but the underlying pathogenic mechanisms are unclear. Using human stem cell models of FTD due to MAPT mutations, we find that tau becomes hyperphosphorylated and mislocalises to neuronal cell bodies and dendrites in cortical neurons, recapitulating a key early event in FTD. Mislocalised tau in the cell body leads to abnormal microtubule dynamics in FTD-MAPT neurons that grossly deform the nuclear membrane, resulting in defective nucleocytoplasmic transport. Neurons in the post-mortem human FTD-MAPT cortex have a high incidence of nuclear deformation, indicating that tau-mediated nuclear membrane dysfunction is an important pathogenic process in FTD. Defects in nucleocytoplasmic transport in FTD point to important commonalities in the pathogenic mechanisms of both tau-mediated dementias and ALS-FTD due to TDP-43 and C9orf72 mutations. 


\section{INTRODUCTION}

The microtubule-associated protein tau (MAPT, tau) is central to the pathogenesis of several different forms of dementia, including Alzheimer's disease (AD), progressive supranuclear palsy, Pick's disease, corticobasal degeneration and frontotemporal dementia (FTD) (Lee et al., 2001; Spillantini and Goedert, 2013). Frontotemporal dementia is the third most common cause of dementia, after Alzheimer's disease and vascular dementia (Rossor et al., 2010). Autosomal dominant missense and splicing mutations in MAPT are causes of inherited or familial frontotemporal dementia (FTDMAPT) (D'Souza et al., 1999; Goedert et al., 2012; Hutton et al., 1998). However, while it is well established that these mutations lead to hyperphosphorylation and aggregation of tau protein in vivo (Ballatore et al., 2007; Goedert et al., 2012), the cell biology of neuronal dysfunction and progressive neurodegeneration in this condition are currently not fully understood.

In healthy neurons, tau protein is almost exclusively localised to the axon, and several mechanisms have been suggested for its highly polarised cellular localisation, including selective mRNA and protein transport, local translation and local degradation (Y. Wang and Mandelkow, 2015). Mislocalisation and aggregation of tau in neuronal cell bodies are common features of tau-mediated dementias, including FTD and AD (Fu et al., 2016; Thies and Mandelkow, 2007; Zempel and Mandelkow, 2015). Protein aggregation is widely considered as inherently pathogenic in neurodegeneration (Fitzpatrick et al., 2017; Hernández-Vega et al., 2017), altering many cellular functions, most notably autophagy and proteostasis (Bence et al., 2001; Caballero et al., 2018; Lim and Yue, 
2015). However, how MAPT mutations lead to tau hyperphosphorylation and mislocalisation, the effects of this mislocalisation on neuronal cell biology, and how this contributes to neuronal dysfunction and neurodegeneration, all remain poorly understood.

As a typical microtubule-binding protein, tau has several roles in regulating microtubule function and intracellular transport (Y. Wang and Mandelkow, 2015). Tau binds both alpha and beta tubulin subunits of microtubules, and has been demonstrated to both stabilise and promote microtubule growth (Kadavath et al., 2015; Witman et al., 1976). The presence of tau on microtubules can alter directions and rates of axonal transport (Dixit et al., 2008; Trinczek et al., 1999). Tau is a natively disordered protein and has recently been found to undergo fluid phase transitions at higher concentrations, nucleating microtubules when it does so (Hernández-Vega et al., 2017). Therefore, it is likely that the changes in tau levels, post-translational modifications and cellular localisation that occur in dementia lead to alterations in microtubule biology, particularly in the neuronal cell body.

To address the question of how MAPT mutations lead to neuronal dysfunction and neurodegeneration, we investigated the effects of two different classes of MAPT mutations on the cell biology of human iPSC-derived cortical neurons. We find that both missense and splicing MAPT mutations cause mislocalisation of tau to the cell bodies of neurons and marked changes in microtubule dynamics. Microtubules in the cell bodies of FTD-MAPT neurons actively deform the nuclear membrane, disrupting 
nucleocytoplasmic transport. Deficits in nuclear envelope function, including nucleocytoplasmic transport, are an important pathological process in ALS-FTD due to repeat expansions in C9orf72 and TDP-43 mutations (Chou et al., 2018; K. Zhang et al., 2018; 2015; Y.-J. Zhang et al., 2016). Our findings demonstrate that dysfunction of the nuclear membrane due to altered microtubule dynamics is a pathogenic process in dementias involving tau, expanding the group of neurodegenerative diseases that involve disrupted nucleocytoplasmic transport and suggesting common mechanisms of neuronal dysfunctional in these heterogeneous conditions. 


\section{RESULTS}

\section{Increased phosphorylation and altered cellular localisation of tau in FTD-MAPT}

\section{neurons}

To study the effects on neuronal cell biology of FTD-MAPT mutations, we generated excitatory cortical neurons (Shi et al., 2012b) from induced pluripotent stem cells (iPSC) derived from individuals with different autosomal dominant mutations in MAPT that are causal for early-onset FTD (Figs. 1 \& S1). We studied two different types of mutations: the MAPT IVS10+16 autosomal dominant mutation, which increases inclusion of exon 10 , encoding the second microtubule-binding repeat, and thus altering the ratio of three (3R) and four (4R) tau isoforms (Hutton et al., 1998; Sposito et al., 2015); and the autosomal dominant MAPT P301L missense mutation that produces an aggregationprone form of tau (Y. Wang and Mandelkow, 2015) (Fig. S1).

Total tau content was similar in neurons of each genotype, collectively referred to here as FTD-MAPT neurons (Fig. 1A). However, tau phosphorylation was increased in FTDMAPT neurons compared to controls (Fig. 1A), notably at Ser404 and Ser202/Thr205 (AT8), epitopes typically hyper-phosphorylated in tau-mediated dementias (Alonso et al., 2004; J.-Z. Wang et al., 2013). As both mutations are dependent on expression of exon 10 of MAPT, we confirmed translation of exon 10 in neurons generated from all iPSC lines by western blotting and mass spectrometry (Fig. 1B, C \& S1). 
Mislocalisation of tau from axons to neuronal cell bodies and dendrites is an early event in FTD in vivo (Götz et al., 1995; Hoover et al., 2010; Kowall and Kosik, 1987). As expected, control neurons showed a predominantly axonal distribution of tau, with tau largely absent from MAP2-positive neuronal cell bodies and dendrites (Fig. 1D). In contrast, tau was commonly present in MAP2-positive cell bodies and dendrites in both MAPT IVS10+16 and P301L neurons (Fig. 1D, E). Furthermore, tau within cell bodies and dendrites of FTD-MAPT neurons was hyperphosphorylated, as detected by AT8immunoreactivity (phospho-S202/T205; Fig. 1D).

\section{Microtubules invade the nucleus in FTD-MAPT neurons}

Given the mislocalisation of tau to the cell bodies of FTD-MAPT neurons, we studied neuronal microtubule dynamics in control and FTD-MAPT neurons (Figs. 2 \& S2). Actively extending microtubules were live-imaged in iPSC-derived neurons of each genotype by expression of GFP-tagged EB3 (Fig. 2; MovieS1), the microtubule plusend binding protein (+TIP; (Akhmanova and Steinmetz, 2008)). Average microtubule dynamics were not different between non-demented control and FTD-MAPT neurons, with similar rates of extensions and retractions measured among the various genotypes (Table S1).

However, microtubule dynamics were qualitatively different in the cell bodies of control neurons compared with FTD-MAPT neurons. Non-demented control neurons typically had many actively growing microtubules within the cell body that extended around a smooth, oval nucleus (Fig. 2A, B; Movie S1). In contrast, many FTD-MAPT neurons 
had microtubules with plus ends projecting into the nucleus (15/26 FTD-MAPT neurons;

Fig. 2), an event that was infrequently detected in both groups of control neurons (2/19;

Fig. 2). Notably, those microtubules that abnormally projected into the nucleus in FTDMAPT neurons frequently originated from a pronounced focus that resembled a microtubule organising centre (Fig. 2A, B; Movie S1).

\section{Microtubules deform the nuclear envelope in FTD-MAPT neurons}

Microtubules couple to the nuclear membrane through the LINC complex (Crisp et al., 2006). This physical association results in transmission of mechanical forces that influence nuclear shape and integrity (Chang et al., 2015), affecting the function of the nuclear envelope (Webster et al., 2009). Given the abnormal projection of microtubules into the nucleus in FTD-MAPT neurons, we studied the shape of the nuclear envelope in iPSC-derived neurons. Marked differences were present in nuclear shape between non-demented controls and FTD-MAPT neurons, as demonstrated by large folds, or invaginations, of the laminB1-positive inner nuclear lamina within the nucleus (Fig. 3). Quantification of the proportions of neurons with deformation of the nuclear membrane, as defined by the presence of LaminB1-positive domains within the nucleus (Fig. 3B; see Fig S3, Methods and Supplementary Materials for details of quantification), found that approximately $25 \%$ of MAPT P301L and $40 \%$ of MAPT IVS $10+16$ neurons have deep nuclear invaginations, compared with less than $10 \%$ of control neurons (Fig. 3A, B). 
Confirming that microtubules actively deformed the nucleus in FTD-MAPT neurons, we found that acute microtubule depolymerisation (with the small molecule nocodazole) reduced the number of nuclear invaginations and restored round nuclear morphology (Fig. 3C, D). We conclude that the pronounced deformations of the neuronal nuclear membrane in FTD-MAPT neurons are actively mediated by microtubules.

\section{Super-resolution imaging demonstrates close apposition of tau and tubulin within nuclear lamina invaginations}

To further study the spatial relationships between tau, microtubules and the nuclear envelope, we conducted a detailed analysis of the neuronal nucleus in iPSC-derived neurons using 3D-STED super-resolution imaging. 3D-STED imaging demonstrated that invaginations of the nuclear lamina present in FTD-MAPT neurons commonly extended deeply into the nucleus, in some cases traversing the entire length of the nucleus, forming pronounced folds (Fig. 4A). In comparison, nuclei from non-demented controls had a regular, smooth morphology, with few examples of nuclear lamina invaginations (Fig. 4A).

As observed by confocal microscopy, tau protein was found to be abundant in the cell bodies of FTD-MAPT neurons by STED imaging, and in those neurons was closely apposed to the outer nuclear membrane (Fig. 4B). Both tau protein and neuronal tubulin were found within nuclear lamina invaginations in FTD-MAPT neurons (Fig. 4B). STED imaging demonstrated that tau within nuclear membrane invaginations is within 100s of nanometres of the nuclear lamina (Fig. 4C). Given that laminB1 filaments line 
the inner surface of the nuclear envelope, which is typically of the order of 15-60nm in width (Burke and Stewart, 2013; Gerace and Huber, 2012), we conclude that tau is in close proximity to proteins in the outer membrane of the nuclear envelope in FTD-MAPT neurons.

Tau-containing nuclear lamina invaginations in neurons of the post-mortem FTD-

\section{MAPT cerebral cortex}

Having identified nuclear lamina defects in iPSC-derived FTD-MAPT neurons, we asked whether alterations of the nuclear lamina are also a feature of FTD-MAPT in vivo. To do so, we studied the incidence of invaginations of the nuclear lamina in the frontal and temporal cortex of two individuals diagnosed with FTD due to MAPT IVS10+16 mutations. We quantified the fraction of all nuclei with nuclear invaginations, in both neuronal and non-neuronal cells, within each brain region. Invaginations of the nuclear lamina, with intranuclear inclusions of laminB1, were found in both non-demented control individuals, but were more frequent in cells of the deep cortical layers in postmortem cerebral cortex from individuals with FTD due to the MAPT IVS10+16 mutation (Fig. 5A, B; Fig. S4). This was the case in both frontal and temporal cortex (Fig. 5B).

Our data from iPSC-derived FTD-MAPT neurons predicted that laminB1-positive nuclear invaginations would be associated with the presence of phosphorylated tau within the neuronal cell body. Consistent with this, we found that the nuclear lamina was grossly disrupted in neurons that had high levels of hyperphosphorylated (AT8+) tau and neurofibrillary tangles in the post-mortem FTD-MAPT IVS10+16 cerebral cortex 
(Fig. 5C, F; Fig. S4), and those neurons frequently contained pronounced nuclear lamina invaginations (Fig. 5C). Furthermore, nuclear lamina invaginations in such neurons also commonly contained AT8-positive, hyperphosphorylated tau (Fig. 5C).

\section{Disrupted nucleocytoplasmic transport in FTD-MAPT neurons}

Abnormalities of the nuclear lamina such as those reported here are also found in ageing diseases, such as Hutchinson-Gilford progeria syndrome (Broers et al., 2006). Nuclear membrane distortion in response to mechanical forces leads to deleterious effects on many aspects of nuclear function, disrupting nucleocytoplasmic transport (Kelley et al., 2011). We confirmed that the nuclear lamina/membrane invaginations present in iPSC-derived FTD-MAPT neurons also contained nuclear pores within these membrane folds, with nuclear pores (labelled by NUP98) co-localising with laminB1positive invaginations (Fig. 6A).

To assess whether alterations in the nuclear membrane in FTD-MAPT neurons result in defects in nucleocytoplasmic transport, we expressed NES:GFP and NLS:RFP from a single construct in iPSC-derived neurons (Mertens et al., 2015). This assay enables measurement of the integrity of both nuclear localisation/accumulation and cytoplasmic retention/nuclear exclusion within individual neurons (Fig. 6B \& S5). Control iPSCderived neurons had discrete cellular distributions of each protein, with prominent nuclear RFP and cytosolic GFP (Fig. 6B). In contrast, localisation of NLS:RFP was altered in FTD-MAPT neurons such that there was a marked decrease in the nuclear:cytoplasmic RFP ratio (Fig. 6B, C). Conversely, nuclear exclusion of NES:GFP 
was reduced in FTD-MAPT neurons, with an increase of GFP within the nucleus (Fig.

6B, C). Together, these data demonstrate defects in the selective permeability of the nuclear envelope in FTD-MAPT neurons, indicating a general failure of nucleocytoplasmic transport within FTD-MAPT neurons. 


\section{DISCUSSION}

The cell and molecular biology of the pathogenesis of frontotemporal dementia due to MAPT mutations are not well understood. Currently, it is thought that MAPT mutations all lead to tau protein aggregation, and that protein aggregation is the primary driver of neurodegeneration (Ballatore et al., 2007; Spillantini and Goedert, 2013). However, protein aggregation may only represent the late stage of the disease, and the processes preceding and leading to neurofibrillary tangle formation and cellular dysfunction remain to be elucidated. Here we report the use of human stem cell systems, combined with MAPT mutations, to study the effects of those mutations on neuronal cell biology, finding that tau-mediated dementias lead to defective neuronal nucleocytoplasmic transport.

Focusing on two different types of MAPT mutations causal for FTD, we have found that both IVS10+16 and P301L mutations lead to marked defects in nucleocytoplasmic transport in human neurons. We find that both missense and splicing mutations in MAPT alter tau protein localisation and phosphorylation within iPSC-derived neurons within four months in cell culture, recapitulating a well-described aspect of early FTD pathology in vivo (Götz et al., 1995; Hoover et al., 2010; Kowall and Kosik, 1987). Mislocalisation of tau in the cell bodies of FTD-MAPT neurons in culture leads to marked changes in microtubule dynamics, causing deformation of the nuclear membrane both in cell culture and in the human FTD-MAPT cortex in vivo. Disruption of the nuclear lamina is commonly associated with dysfunction of the nuclear envelope, and we find marked disruption of nucleocytoplasmic transport in FTD-MAPT neurons. 
Together, these data indicate that perturbation of the function of the nuclear membrane and disruption of nucleocytoplasmic transport is an important pathological process in FTD due to MAPT mutations.

An early event in FTD is the mislocalisation of tau from axons to cell bodies and dendrites, and this key stage in disease progression is also an early event in PSCderived models of FTD-MAPT. In vivo, mislocalisation of tau is typically associated with tau hyperphosphorylation (Götz et al., 1995; Spillantini and Goedert, 2013). We find this also occurs in iPSC-derived FTD-MAPT neurons, where we detected increased tau phosphorylation at serine 202/threonine 205 (the AT8 epitope) and also at serine 404 . The sequence in which mislocalisation and hyperphosphorylation take place in FTD in vivo, and in iPSC-derived FTD-MAPT neurons in culture, is not currently clear, nor are the mechanisms by which these processes occur. The appearance of tau within cell bodies and dendrites indicates a breakdown of the cellular polarity mechanisms that maintain the axonal enrichment of tau protein and its exclusion from the somatodendritic compartment, mechanisms which are poorly understood.

The two heterozygous, dominant MAPT mutations studied here have different effects on tau protein in neurons. The MAPT P301L missense mutation, like many missense mutations in the microtubule binding region domain of MAPT, increases the tendency of tau to aggregate in cell-free assays and in transgenic mouse models (Bergen et al., 2005; Lewis et al., 2000; Shammas et al., 2015). In contrast, the IVS10+16 mutation is not a coding mutation, but rather is an intronic single base change that favours the 
inclusion of exon 10 in the MAPT mRNA, increasing the amount of tau containing four microtubule-binding repeats $(4 R)$, relative to the three-repeat $(3 R)$ form (Hutton et al., 1998). However, despite these differences, the changes in the forms of tau in both MAPT P301L and IVS10+16 neurons both lead to mislocalisation and increased phosphorylation of tau. This finding suggests that either the presence of a pool of P301L tau, or a shift in the $3 \mathrm{R}: 4 \mathrm{R}$ tau ratio, alter a common pathway that regulates tau distribution within neurons, tau phosphorylation, or both.

In both MAPT IVS 10+16 and P301L mutant neurons, the appearance of tau in cell bodies is accompanied by marked qualitative changes in neuronal microtubule dynamics. Microtubules in FTD-MAPT neurons actively deform the nuclear envelope, which we find can be reversed by depolymerisation of microtubules. Tau has multiple roles in stabilising microtubules (Y. Wang and Mandelkow, 2015), and microtubules are coupled to the nuclear membrane through the LINC complex (Chang et al., 2015; Crisp et al., 2006; Luo et al., 2016). Therefore, it is likely that the overall effect of the presence of tau in the cell body is to promote microtubule stability, leading to increased pushing forces on the nuclear membrane and the formation of invaginations in the nuclear membrane. As tau has recently also been found to promote microtubule nucleation when undergoing phase transitions at high concentration (Hernández-Vega et al., 2017), accumulation of tau in the cell body may also lead to a net increase in microtubule growth events and increased pushing forces on the nucleus by facilitating microtubule nucleation. 
Alterations in nuclear shape and nuclear membrane function are a common feature of cellular ageing, including in the nervous system, and are associated with multiple deleterious changes in nuclear biology, including chromatin changes and disrupted nucleocytoplasmic transport (Frost, 2016; Oberdoerffer and Sinclair, 2007). Drosophila models of FTD, with neuronal expression of human MAPT R406W, have nuclear shape abnormalities and chromatin changes (Frost et al., 2016). Perturbations of the nuclear lamina have been described in the post-mortem AD brain (Frost et al., 2016), including the juxtaposition of neurofibrillary tangles of tau with the nuclear membrane (Sheffield et al., 2006). We also find here an increase in nuclear invaginations in neurons of the human post-mortem MAPT IVS10+16 cortex, and the presence of hyperphosphorylated tau within nuclear invaginations in tangle-bearing neurons. Together, these different studies are consistent with a pathological effect of tau within the neuronal cell body in FTD and AD, where the presence of tau alters microtubule biology, resulting in pronounced abnormalities of the neuronal nucleus and defective nucleocytoplasmic transport.

Microtubule deformation of the nucleus is a phenotype also seen in classic laminopathies such as the accelerated aging disorder Hutchinson-Gilford Progeria Syndrome (HGPS), where the primary defect is due to mutant lamin A/C protein (Capell and Collins, 2006). In that case, microtubules also contribute to nuclear deformations, leading to defects in nucleocytoplasmic transport (Kelley et al., 2011; Larrieu et al., 2014; Snow et al., 2013). Changes in nuclear envelope function in other neurodegenerative diseases, including ALS-FTD due to repeat expansions in C9orf72 
and Huntington's disease, have recently been reported (Freibaum et al., 2015; Grima et al., 2017; Jovičić et al., 2015; K. Zhang et al., 2015). Our finding here of disruption of the neuronal nuclear membrane as a consequence of MAPT mutations in frontotemporal dementia extends this pathogenic mechanism to dementias where protein aggregation has been thought to be the primary driver of neurodegeneration. These data suggest that dysfunction of the nuclear membrane may be a common pathogenic process in diverse neurodegenerative diseases, which could be targeted therapeutically with agents that regulate microtubule functions, nucleocytoplasmic transport and/or associated processes. 


\section{METHODS}

\section{Generation of iPSC-derived cortical neurons and drug treatments}

MAPT IVS10+16-A and MAPT IVS10+16-B mutant iPSCs were as reported in (Sposito et al., 2015). MAPT P301L was generated from Janssen Pharmaceutica NV by TALEN editing the line MAPT P301-isogenic, under the IMI STEMBANCC project agreement ICD 483960. The non-demented control line was previously reported (Israel et al., 2012). Pluripotent cells were cultured as previously described (Beers et al., 2012). Differentiation of iPSCs to cortical neurons was carried out as described, with minor modifications (Shi et al., 2012b; 2012a). For nocodazole (Tocris) treatment, neurons were grown for 120 days in vitro (DIV) and compound was added at $33 \mu \mathrm{M}$ for $4 \mathrm{hr}$ before imaging.

\section{Protein extraction and western blot analysis}

Total cell protein was extracted using RIPA buffer (Sigma; R0278) supplemented with protease inhibitors (Sigma; 4693159001) and Halt phosphatase inhibitors (Thermo Scientific; 78420). Immunoblots were detected using LI-COR Odyssey CLx Infrared Imaging System and processed with the Image Studio Software (LI-COR).

\section{Immunoprecipitation and mass spectrometric analysis of intracellular tau}

Tau was immunoprecipitated from $1 \mathrm{mg}$ of total protein extracted from iPSC-derived neurons (120 DIV) using a polyclonal anti-tau antibody (Dako Cytomation; A0024). Immunoprecipitated samples were analysed by western blot using a monoclonal tau antibody (MN1000; ThermoFisher Scientific) or stained with colloidal blue (Invitrogen; 
LC6025). Bands that corresponded to tau by western blot analysis were excised from the colloidal blue SDS-PAGE. Peptide masses of digested protein samples were determined using a Bruker ultrafleXtreme Maldi mass spectrometer in reflectron mode and $\mathrm{ms} / \mathrm{ms}$ fragmentation performed in LIFT mode. Data analysis was with FlexAnalysis, BioTools and ProteinScape software (Bruker). Database searches of the combined mass fingerprint-ms/ms data were performed using Mascot (http://www.matrixscience.com).

\section{Confocal and 3D-STED microscopy and image analysis}

Standard confocal images of fixed and immunostained cells were acquired with an Olympus Inverted FV1000 confocal microscope and processed using Fiji software (Schindelin et al., 2012). 3D-STED imaging was performed on a custom built, dual color, beam scanning system with gated detection optically identical to the instrument described in (Bottanelli et al., 2016). For image analysis of colocalisation of tau and MAP2, Pearson's R correlation was calculated using the Coloc2 plugin for Fiji software (https://imagej.net/Coloc2). To quantify nuclear invaginations, at least 5 imaging fields from three independent experiments for genotype were analysed using a custom plugin for the Fiji. See Fig S3 for details.

\section{Live imaging of microtubule dynamics}

Neurons were grown to 120 DIV in individual $\mu$-Dish $35 \mathrm{~mm}$ dishes (Ibidi; 80136) and transfected with a plasmid encoding for GFP-EB3 (Addgene plasmid \# 56474). 48 hours after transfection, neurons were imaged live using a Leica SP5 microscope equipped 
with a controlled environment chamber (37 C; $\left.5 \% \mathrm{CO}_{2}\right)$. Images were acquired at resonant scanning with a $63 x$ objective ( 1 frame/sec). Movies were analysed using the plusTipTracker software (Applegate et al., 2011).

\section{Analysis of human post-mortem cerebral cortex}

Human brain sections were obtained from the Queen's Square Brain Bank, Institute of Neurology, University College London. Control brains included one male (age 71) and one female (age 56). FTD-MAPT IVS10+16 brains were from two males (age 52 and 66). Nuclear lamina invaginations were quantified after 3,3'-Diaminobenzidine (DAB) staining of LaminB1. Nuclei were scored from 20 randomly acquired imaging fields, from each post-mortem sample, by three observers blinded to sample identity. The percentage of nuclei with invaginations relative to total nuclei was calculated for each imaging field (see Fig. S4C). For fluorescent immunostaining of laminB1 and tau, following incubation with secondary antibodies and DAPI nuclear counterstain, images were acquired using an Olympus Inverted FV1000 confocal microscope .

\section{Nucleocytoplasmic transport assay}

Nucleocytoplasmic trafficking was analysed by infection of 120 DIV human iPSCderived neurons with the pLVX-EF1alpha-2xGFP:NES-IRES-2xRFP:NLS construct (Addgene \#71396; Mertens et al., 2015). After 6 days, neurons were fixed and immunostained for $\beta 3$-tubulin and GFP. Only cells positive for neuron-specific $\beta 3$-tubulin were considered. The nuclear to cytoplasmic ratios of both GFP and RFP 
bioRxiv preprint doi: https://doi.org/10.1101/328187; this version posted May 22, 2018. The copyright holder for this preprint (which was not certified by peer review) is the author/funder, who has granted bioRxiv a license to display the preprint in perpetuity. It is made available under aCC-BY 4.0 International license.

(nucRFP:cytRFP and nucGFP:cytGFP) were calculated separately using the integrated density of ROIs drawn within and outside the nucleus (see Fig.S5 for details). 


\section{ACKNOWLEDGEMENTS}

FJL's group is supported by a Wellcome Trust Senior Investigator Award, the Alborada Trust's funding of the Alzheimer's Research UK Stem Cell Research Centre and the IMI program StemBANCC. SPJ is a Wellcome Trust Senior Investigator. SW and JH received funding from the National Institute for Health Research University College London Hospitals Biomedical Research Centre and SW by an ARUK Senior Research Fellowship (ARUK-SRF2016B-2). Research in SPJ and FJL's groups benefits from core support to the Gurdon Institute from the Wellcome Trust and Cancer Research UK. The research leading to these results has received support from the Innovative Medicines Initiative Joint Undertaking under grant agreement $n^{\circ} 115439$, resources of which are composed of financial contribution from the European Union's Seventh Framework Programme (FP7/20072013) and EFPIA companies' in-kind contribution. This report reflects only the author's views and neither the IMI JU nor EFPIA nor the European Commission are liable for any use that may be made of the information contained therein. 


\section{REFERENCES}

Akhmanova, A., Steinmetz, M.O., 2008. Tracking the ends: a dynamic protein network controls the fate of microtubule tips. Nat. Rev. Mol. Cell Biol. 9, 309-322. doi:10.1038/nrm2369

Alonso, A.D.C., Mederlyova, A., Novak, M., Grundke-lqbal, I., Iqbal, K., 2004. Promotion of hyperphosphorylation by frontotemporal dementia tau mutations. J. Biol. Chem. 279, 34873-34881. doi:10.1074/jbc.M405131200

Applegate, K.T., Besson, S., Matov, A., Bagonis, M.H., Jaqaman, K., Danuser, G., 2011. plusTipTracker: Quantitative image analysis software for the measurement of microtubule dynamics. J. Struct. Biol. 176, 168-184. doi:10.1016/j.jsb.2011.07.009 Ballatore, C., Lee, V.M.-Y., Trojanowski, J.Q., 2007. Tau-mediated neurodegeneration in Alzheimer's disease and related disorders. Nat. Rev. Neurosci. 8, 663-672. doi:10.1038/nrn2194

Beers, J., Gulbranson, D.R., George, N., Siniscalchi, L.I., Jones, J., Thomson, J.A., Chen, G., 2012. Passaging and colony expansion of human pluripotent stem cells by enzyme-free dissociation in chemically defined culture conditions. Nat Protoc 7 , 2029-2040. doi:10.1038/nprot.2012.130

Bence, N.F., Sampat, R.M., Kopito, R.R., 2001. Impairment of the ubiquitin-proteasome system by protein aggregation. Science $292,1552-1555$.

doi:10.1126/science.292.5521.1552

Bergen, von, M., Barghorn, S., Biernat, J., Mandelkow, E.-M., Mandelkow, E., 2005.

Tau aggregation is driven by a transition from random coil to beta sheet structure. Biochim. Biophys. Acta 1739, 158-166. doi:10.1016/j.bbadis.2004.09.010 
Bottanelli, F., Kromann, E.B., Allgeyer, E.S., Erdmann, R.S., Wood Baguley, S.,

Sirinakis, G., Schepartz, A., Baddeley, D., Toomre, D.K., Rothman, J.E.,

Bewersdorf, J., 2016. Two-colour live-cell nanoscale imaging of intracellular targets.

Nat Commun 7, 10778. doi:10.1038/ncomms10778

Broers, J.L.V., Ramaekers, F.C.S., Bonne, G., Yaou, R.B., Hutchison, C.J., 2006.

Nuclear lamins: laminopathies and their role in premature ageing. Physiol. Rev. 86, 967-1008. doi:10.1152/physrev.00047.2005

Burke, B., Stewart, C.L., 2013. The nuclear lamins: flexibility in function. Nat. Rev. Mol.

Cell Biol. 14, 13-24. doi:10.1038/nrm3488

Caballero, B., Wang, Y., Diaz, A., Tasset, I., Juste, Y.R., Stiller, B., Mandelkow, E.-M.,

Mandelkow, E., Cuervo, A.M., 2018. Interplay of pathogenic forms of human tau

with different autophagic pathways. Aging Cell 17, e12692. doi:10.1111/acel.12692

Capell, B.C., Collins, F.S., 2006. Human laminopathies: nuclei gone genetically awry.

Nat. Rev. Genet. 7, 940-952. doi:10.1038/nrg1906

Chang, W., Worman, H.J., Gundersen, G.G., 2015. Accessorizing and anchoring the LINC complex for multifunctionality. J. Cell Biol. 208, 11-22.

doi:10.1083/jcb.201409047

Chou, C.-C., Zhang, Y., Umoh, M.E., Vaughan, S.W., Lorenzini, I., Liu, F., Sayegh, M., Donlin-Asp, P.G., Chen, Y.H., Duong, D.M., Seyfried, N.T., Powers, M.A., Kukar, T., Hales, C.M., Gearing, M., Cairns, N.J., Boylan, K.B., Dickson, D.W., Rademakers, R., Zhang, Y.-J., Petrucelli, L., Sattler, R., Zarnescu, D.C., Glass, J.D., Rossoll, W., 2018. TDP-43 pathology disrupts nuclear pore complexes and nucleocytoplasmic transport in ALS/FTD. Nature Neuroscience 21, 228-239. doi:10.1038/s41593-017- 


\section{$0047-3$}

Crisp, M., Liu, Q., Roux, K., Rattner, J.B., Shanahan, C., Burke, B., Stahl, P.D., Hodzic,

D., 2006. Coupling of the nucleus and cytoplasm. J. Cell Biol. 172, 41-53.

doi:10.1083/jcb.200509124

D'Souza, I., Poorkaj, P., Hong, M., Nochlin, D., Lee, V.M., Bird, T.D., Schellenberg,

G.D., 1999. Missense and silent tau gene mutations cause frontotemporal dementia with parkinsonism-chromosome 17 type, by affecting multiple alternative RNA splicing regulatory elements. PNAS 96, 5598-5603.

Dixit, R., Ross, J.L., Goldman, Y.E., Holzbaur, E.L.F., 2008. Differential regulation of dynein and kinesin motor proteins by tau. Science 319, 1086-1089. doi:10.1126/science.1152993

Fitzpatrick, A.W.P., Falcon, B., He, S., Murzin, A.G., Murshudov, G., Garringer, H.J., Crowther, R.A., Ghetti, B., Goedert, M., Scheres, S.H.W., 2017. Cryo-EM structures of tau filaments from Alzheimer's disease. Nature 547, 185-190.

doi:10.1038/nature23002

Freibaum, B.D., Lu, Y., Lopez-Gonzalez, R., Kim, N.C., Almeida, S., Lee, K.-H., Badders, N., Valentine, M., Miller, B.L., Wong, P.C., Petrucelli, L., Kim, H.J., Gao, F.-B., Taylor, J.P., 2015. GGGGCC repeat expansion in $<i>C 9 o r f 72</ i>$ compromises nucleocytoplasmic transport. Nature 525, 129-133. doi:10.1038/nature14974

Frost, B., 2016. Alzheimer's disease: An acquired neurodegenerative laminopathy.

Nucleus 7, 275-283. doi:10.1080/19491034.2016.1183859

Frost, B., Bardai, F.H., Feany, M.B., 2016. Lamin Dysfunction Mediates 
Neurodegeneration in Tauopathies. Curr. Biol. 26, 129-136.

doi:10.1016/j.cub.2015.11.039

Fu, H., Hussaini, S.A., Wegmann, S., Profaci, C., Daniels, J.D., Herman, M., Emrani, S., Figueroa, H.Y., Hyman, B.T., Davies, P., Duff, K.E., 2016. 3D Visualization of the Temporal and Spatial Spread of Tau Pathology Reveals Extensive Sites of Tau Accumulation Associated with Neuronal Loss and Recognition Memory Deficit in Aged Tau Transgenic Mice. PLoS ONE 11, e0159463.

doi:10.1371/journal.pone.0159463

Gerace, L., Huber, M.D., 2012. Nuclear lamina at the crossroads of the cytoplasm and nucleus. J. Struct. Biol. 177, 24-31. doi:10.1016/j.jsb.2011.11.007

Goedert, M., Ghetti, B., Spillantini, M.G., 2012. Frontotemporal dementia: implications for understanding Alzheimer disease. Cold Spring Harbor Perspectives in Medicine 2, a006254-a006254. doi:10.1101/cshperspect.a006254

Götz, J., Probst, A., Spillantini, M.G., Schäfer, T., Jakes, R., Bürki, K., Goedert, M., 1995. Somatodendritic localization and hyperphosphorylation of tau protein in transgenic mice expressing the longest human brain tau isoform. EMBO J. 14, 1304-1313.

Grima, J.C., Daigle, J.G., Arbez, N., Cunningham, K.C., Zhang, K., Ochaba, J., Geater, C., Morozko, E., Stocksdale, J., Glatzer, J.C., Pham, J.T., Ahmed, I., Peng, Q., Wadhwa, H., Pletnikova, O., Troncoso, J.C., Duan, W., Snyder, S.H., Ranum, L.P.W., Thompson, L.M., Lloyd, T.E., Ross, C.A., Rothstein, J.D., 2017. Mutant Huntingtin Disrupts the Nuclear Pore Complex. Neuron 94, 93-107.e6. doi:10.1016/j.neuron.2017.03.023 
Hernández-Vega, A., Braun, M., Scharrel, L., Jahnel, M., Wegmann, S., Hyman, B.T., Alberti, S., Diez, S., Hyman, A.A., 2017. Local Nucleation of Microtubule Bundles through Tubulin Concentration into a Condensed Tau Phase. Cell Rep 20, 23042312. doi:10.1016/j.celrep.2017.08.042

Hoover, B.R., Reed, M.N., Su, J., Penrod, R.D., Kotilinek, L.A., Grant, M.K., Pitstick, R., Carlson, G.A., Lanier, L.M., Yuan, L.-L., Ashe, K.H., Liao, D., 2010. Tau mislocalization to dendritic spines mediates synaptic dysfunction independently of neurodegeneration. Neuron 68, 1067-1081. doi:10.1016/j.neuron.2010.11.030 Hutton, M., Lendon, C.L., Rizzu, P., Baker, M., Froelich, S., Houlden, H., PickeringBrown, S., Chakraverty, S., Isaacs, A., Grover, A., Hackett, J., Adamson, J., Lincoln, S., Dickson, D., Davies, P., Petersen, R.C., Stevens, M., de Graaff, E., Wauters, E., van Baren, J., Hillebrand, M., Joosse, M., Kwon, J.M., Nowotny, P., Che, L.K., Norton, J., Morris, J.C., Reed, L.A., Trojanowski, J., Basun, H., Lannfelt, L., Neystat, M., Fahn, S., Dark, F., Tannenberg, T., Dodd, P.R., Hayward, N., Kwok, J.B., Schofield, P.R., Andreadis, A., Snowden, J., Craufurd, D., Neary, D., Owen, F., Oostra, B.A., Hardy, J., Goate, A., van Swieten, J., Mann, D., Lynch, T., Heutink, P., 1998. Association of missense and 5'-splice-site mutations in tau with the inherited dementia FTDP-17. Nature 393, 702-705. doi:10.1038/31508 Israel, M.A., Yuan, S.H., Bardy, C., Reyna, S.M., Mu, Y., Herrera, C., Hefferan, M.P., Van Gorp, S., Nazor, K.L., Boscolo, F.S., Carson, C.T., Laurent, L.C., Marsala, M., Gage, F.H., Remes, A.M., Koo, E.H., Goldstein, L.S.B., 2012. Probing sporadic and familial Alzheimer's disease using induced pluripotent stem cells. Nature 482, 216220. doi:10.1038/nature10821 
Jovičić, A., Mertens, J., Boeynaems, S., Bogaert, E., Chai, N., Yamada, S.B., Paul, J.W., Sun, S., Herdy, J.R., Bieri, G., Kramer, N.J., Gage, F.H., Van Den Bosch, L., Robberecht, W., Gitler, A.D., 2015. Modifiers of C9orf72 dipeptide repeat toxicity connect nucleocytoplasmic transport defects to FTD/ALS. Nature Neuroscience 18, 1226-1229. doi:10.1038/nn.4085

Kadavath, H., Hofele, R.V., Biernat, J., Kumar, S., Tepper, K., Urlaub, H., Mandelkow, E., Zweckstetter, M., 2015. Tau stabilizes microtubules by binding at the interface between tubulin heterodimers. Proc. Natl. Acad. Sci. U.S.A. 112, 7501-7506. doi:10.1073/pnas.1504081112

Kelley, J.B., Datta, S., Snow, C.J., Chatterjee, M., Ni, L., Spencer, A., Yang, C.-S., Cubeñas-Potts, C., Matunis, M.J., Paschal, B.M., 2011. The defective nuclear lamina in Hutchinson-gilford progeria syndrome disrupts the nucleocytoplasmic Ran gradient and inhibits nuclear localization of Ubc9. Mol. Cell. Biol. 31, 3378-3395. doi:10.1128/MCB.05087-11

Kowall, N.W., Kosik, K.S., 1987. Axonal disruption and aberrant localization of tau protein characterize the neuropil pathology of Alzheimer's disease. Annals of Neurology 22, 639-643. doi:10.1002/ana.410220514

Larrieu, D., Britton, S., Demir, M., Rodriguez, R., Jackson, S.P., 2014. Chemical inhibition of NAT10 corrects defects of laminopathic cells. Science 344, 527-532. doi:10.1126/science. 1252651

Lee, V.M., Goedert, M., Trojanowski, J.Q., 2001. Neurodegenerative tauopathies. Annu. Rev. Neurosci. 24, 1121-1159. doi:10.1146/annurev.neuro.24.1.1121 Lewis, J., McGowan, E., Rockwood, J., Melrose, H., Nacharaju, P., Van Slegtenhorst, 
M., Gwinn-Hardy, K., Paul Murphy, M., Baker, M., Yu, X., Duff, K., Hardy, J., Corral, A., Lin, W.L., Yen, S.H., Dickson, D.W., Davies, P., Hutton, M., 2000. Neurofibrillary tangles, amyotrophy and progressive motor disturbance in mice expressing mutant (P301L) tau protein. Nat. Genet. 25, 402-405. doi:10.1038/78078

Lim, J., Yue, Z., 2015. Neuronal Aggregates: Formation, Clearance, and Spreading.

Dev. Cell 32, 491-501. doi:10.1016/j.devcel.2015.02.002

Luo, Y., Lee, I.-W., Jo, Y.-J., Namgoong, S., Kim, N.-H., 2016. Depletion of the LINC complex disrupts cytoskeleton dynamics and meiotic resumption in mouse oocytes.

Scientific Reports 6, 2119. doi:10.1038/srep20408

Mertens, J., Paquola, A.C.M., Ku, M., Hatch, E., Böhnke, L., Ladjevardi, S., McGrath, S., Campbell, B., Lee, H., Herdy, J.R., Gonçalves, J.T., Toda, T., Kim, Y., Winkler, J., Yao, J., Hetzer, M.W., Gage, F.H., 2015. Directly Reprogrammed Human Neurons Retain Aging-Associated Transcriptomic Signatures and Reveal AgeRelated Nucleocytoplasmic Defects. Cell Stem Cell 17, 705-718. doi:10.1016/j.stem.2015.09.001

Oberdoerffer, P., Sinclair, D.A., 2007. The role of nuclear architecture in genomic instability and ageing. Nat. Rev. Mol. Cell Biol. 8, 692-702. doi:10.1038/nrm2238 Rossor, M.N., Fox, N.C., Mummery, C.J., Schott, J.M., Warren, J.D., 2010. The diagnosis of young-onset dementia. The Lancet Neurology 9, 793-806. doi:10.1016/S1474-4422(10)70159-9

Schindelin, J., Arganda-Carreras, I., Frise, E., Kaynig, V., Longair, M., Pietzsch, T., Preibisch, S., Rueden, C., Saalfeld, S., Schmid, B., Tinevez, J.-Y., White, D.J., Hartenstein, V., Eliceiri, K., Tomancak, P., Cardona, A., 2012. Fiji: an open-source 
platform for biological-image analysis. Nat. Methods 9, 676-682.

doi:10.1038/nmeth.2019

Shammas, S.L., Garcia, G.A., Kumar, S., Kjaergaard, M., Horrocks, M.H., Shivji, N.,

Mandelkow, E., Knowles, T.P.J., Mandelkow, E., Klenerman, D., 2015. A

mechanistic model of tau amyloid aggregation based on direct observation of

oligomers. Nat Commun 6, 7025. doi:10.1038/ncomms8025

Sheffield, L.G., Miskiewicz, H.B., Tannenbaum, L.B., Mirra, S.S., 2006. Nuclear pore

complex proteins in Alzheimer disease. J. Neuropathol. Exp. Neurol. 65, 45-54.

Shi, Y., Kirwan, P., Livesey, F.J., 2012a. Directed differentiation of human pluripotent stem cells to cerebral cortex neurons and neural networks. Nat Protoc 7, 18361846. doi:10.1038/nprot.2012.116

Shi, Y., Kirwan, P., Smith, J., Robinson, H.P.C., Livesey, F.J., 2012b. Human cerebral cortex development from pluripotent stem cells to functional excitatory synapses.

Nature Neuroscience 15, 477-86- S1. doi:10.1038/nn.3041

Snow, C.J., Dar, A., Dutta, A., Kehlenbach, R.H., Paschal, B.M., 2013. Defective nuclear import of Tpr in Progeria reflects the Ran sensitivity of large cargo transport. J. Cell Biol. 201, 541-557. doi:10.1083/jcb.201212117

Spillantini, M.G., Goedert, M., 2013. Tau pathology and neurodegeneration. The Lancet Neurology 12, 609-622. doi:10.1016/S1474-4422(13)70090-5

Sposito, T., Preza, E., Mahoney, C.J., Setó-Salvia, N., Ryan, N.S., Morris, H.R., Arber, C., Devine, M.J., Houlden, H., Warner, T.T., Bushell, T.J., Zagnoni, M., Kunath, T., Livesey, F.J., Fox, N.C., Rossor, M.N., Hardy, J., Wray, S., 2015. Developmental regulation of tau splicing is disrupted in stem cell-derived neurons from 
frontotemporal dementia patients with the $10+16$ splice-site mutation in MAPT.

Human Molecular Genetics 24, 5260-5269. doi:10.1093/hmg/ddv246

Thies, E., Mandelkow, E.-M., 2007. Missorting of tau in neurons causes degeneration of synapses that can be rescued by the kinase MARK2/Par-1. J. Neurosci. 27, 28962907. doi:10.1523/JNEUROSCI.4674-06.2007

Trinczek, B., Ebneth, A., Mandelkow, E.M., Mandelkow, E., 1999. Tau regulates the attachment/detachment but not the speed of motors in microtubule-dependent transport of single vesicles and organelles. J. Cell. Sci. 112 ( Pt 14), 2355-2367.

Wang, J.-Z., Xia, Y.-Y., Grundke-lqbal, I., Iqbal, K., 2013. Abnormal hyperphosphorylation of tau: sites, regulation, and molecular mechanism of neurofibrillary degeneration. J. Alzheimers Dis. 33 Suppl 1, S123-39. doi:10.3233/JAD-2012-129031

Wang, Y., Mandelkow, E., 2015. Tau in physiology and pathology. Nat. Rev. Neurosci. doi:doi:10.1038/nrn.2015.1

Webster, M., Witkin, K.L., Cohen-Fix, O., 2009. Sizing up the nucleus: nuclear shape, size and nuclear-envelope assembly. J. Cell. Sci. 122, 1477-1486. doi:10.1242/jcs.037333

Witman, G.B., Cleveland, D.W., Weingarten, M.D., Kirschner, M.W., 1976. Tubulin requires tau for growth onto microtubule initiating sites. PNAS 73, 4070-4074. doi:10.1073/pnas.73.11.4070

Zempel, H., Mandelkow, E.-M., 2015. Tau missorting and spastin-induced microtubule disruption in neurodegeneration: Alzheimer Disease and Hereditary Spastic Paraplegia. Mol Neurodegener 10, 68. doi:10.1186/s13024-015-0064-1 
Zhang, K., Daigle, J.G., Cunningham, K.M., Coyne, A.N., Ruan, K., Grima, J.C., Bowen, K.E., Wadhwa, H., Yang, P., Rigo, F., Taylor, J.P., Gitler, A.D., Rothstein, J.D., Lloyd, T.E., 2018. Stress Granule Assembly Disrupts Nucleocytoplasmic Transport. Cell 173, 958-971.e17. doi:10.1016/j.cell.2018.03.025

Zhang, K., Donnelly, C.J., Haeusler, A.R., Grima, J.C., Machamer, J.B., Steinwald, P., Daley, E.L., Miller, S.J., Cunningham, K.M., Vidensky, S., Gupta, S., Thomas, M.A., Hong, I., Chiu, S.-L., Huganir, R.L., Ostrow, L.W., Matunis, M.J., Wang, J., Sattler, R., Lloyd, T.E., Rothstein, J.D., 2015. The C9orf72 repeat expansion disrupts nucleocytoplasmic transport. Nature 525, 56-61. doi:10.1038/nature14973 Zhang, Y.-J., Gendron, T.F., Grima, J.C., Sasaguri, H., Jansen-West, K., Xu, Y.-F., Katzman, R.B., Gass, J., Murray, M.E., Shinohara, M., Lin, W.-L., Garrett, A., Stankowski, J.N., Daughrity, L., Tong, J., Perkerson, E.A., Yue, M., Chew, J., Castanedes-Casey, M., Kurti, A., Wang, Z.S., Liesinger, A.M., Baker, J.D., Jiang, J., Lagier-Tourenne, C., Edbauer, D., Cleveland, D.W., Rademakers, R., Boylan, K.B., Bu, G., Link, C.D., Dickey, C.A., Rothstein, J.D., Dickson, D.W., Fryer, J.D., Petrucelli, L., 2016. C9ORF72 poly(GA) aggregates sequester and impair HR23 and nucleocytoplasmic transport proteins. Nature Neuroscience 19, 668-677. doi:10.1038/nn.4272 
FIGURE LEGENDS

Figure 1. Increased phosphorylation and altered cellular localisation of tau in FTD-MAPT neurons

(A) Phosphorylated tau (pS404; AT8 [pS202/pT205]) is increased as a fraction of total tau (epitope 243-441) in FTD-MAPT neurons (MAPT IVS10+16-A/B and MAPT P301L) compared to non-demented and MAPT P301 isogenic control neurons (iPSC-derived neurons at 120 DIV; three biological replicates). $\beta$-actin was used as a loading control. Molecular weight $(\mathrm{kDa})$ indicated.

(B) Tau isoforms with three (3R; red) or four (4R; inclusion of region 2; green) microtubule binding regions were detected by western blot analysis of dephosphorylated protein extracts from iPSC-derived control and FTD-MAPT cortical neurons (120 DIV) and from post-mortem human cerebral cortex (non-demented individual). Tau isoforms were identified relative to a commercial tau ladder (Sigma). Molecular weight $(\mathrm{kDa})$ indicated.

(C) Peptide sequences identified by tau IP/mass spectrometry from iPSC-derived cortical neurons, confirming the inclusion of repeat 2 (corresponding to exon 10) of $4 R$ tau. In MAPT P301L neurons, both proline and leucine were identified at position 301 (highlighted red). See also Figure S2.

(D) Tau protein is mislocalised to MAP2-positive cell bodies and dendrites in iPSCderived FTD-MAPT neurons. Confocal images of iPSC-derived control and FTD-MAPT neurons, (120 DIV; tau, green; MAP2, red; DAPI, blue). Hyperphosphorylated, AT8- 
positive tau (AT8; green) is found in cell bodies of FTD-MAPT neurons (arrows), but not in controls ( $\beta 3$-tubulin, red; DAPI, blue). Scale bars $=20 \mu \mathrm{m}$.

(E) Increased co-localisation of tau and MAP2 protein in FTD-MAPT neurons, compared to non-demented control neurons, analysed by Pearson's R correlation (control lines: grey bars; FTD-MAPT lines: black; significance was determined for three sample comparison of non-demented control and two MAPT IVS10+16 lines using one-way ANOVA followed by Tukey's test; ${ }^{*}=\mathrm{P}<0.05,{ }^{* * *}=\mathrm{P}<0.001$. Pair-wise comparison of the MAPT P301L line and its isogenic control was carried out using Student's $t$ test; ${ }^{*}=\mathrm{P}<0.05$; error bar represents s.e.m.; $\mathrm{n}=3$ independent experiments).

See also Figure S1.

Figure 2. Microtubules invade the nucleus in FTD-MAPT neurons

(A) Representative stills (5-second intervals) from GFP-EB3 live imaging of nondemented iPSC-derived control and MAPT P301L neurons (120 DIV) show actively growing microtubules extending into the nucleus in the MAPT mutant neurons. Red arrows indicate the position of an EB3-GFP comet at time point 0 (far left); green arrows indicate the comet position in each subsequent frame. Right panels show cumulative tracks (growth tracks) of EB3 comets over the 200-second interval.

(B) Total microtubule trajectories (200-second interval) demonstrate multiple microtubule extensions into the nuclei of FTD-MAPT neurons (MAPT IVS10+16-A/B and MAPT P301L), compared to non-demented and MAPT P301 isogenic control neurons. Red arrows indicate examples of trajectories within the nucleus. Bars indicate the number of sampled neurons with (black), and without (white), nuclear EB3 growth 
tracks; $n=$ number of imaged neurons. Scale bars $=10 \mu \mathrm{m}$. See also Video $\mathrm{S} 1$ and Table S1.

See also Figure S2.

\section{Figure 3. Microtubules deform the nuclear envelope in FTD-MAPT neurons}

(A) Marked abnormalities of nuclear lamina shape in FTD-MAPT neurons. Confocal images of the nuclear lamina (laminB1, green) in FTD-MAPT neurons (MAPT

IVS10+16-A/B and MAPT P301L; neuronal transcription factor CTIP2, red) compared to non-demented and MAPT P301 isogenic control neurons (120 DIV). White arrows indicate examples of nuclei with pronounced deformation of the nuclear lamina. (B) FTD-MAPT neurons have increased numbers of cells with deformed nuclear membranes, as defined by the shape of the inner nuclear lamina. Cartoon of image analysis method used to quantify nuclear invaginations, as a measure of distortion of the nuclear membrane: nuclear area was established using DAPI (blue), and nuclear lamina (LaminB1; green) was assigned as either nuclear boundary or invaginated (i.e., within the nucleus). The fraction of total laminB1 that was invaginated was used empirically to define a threshold for defining neurons as having nuclear membrane invaginations (see Figure S3 for details). Between 25\% (MAPT P301L) and 40\% (MAPT IVS10+16) of FTD-MAPT neurons have nuclear invaginations, compared with less than $10 \%$ of control neurons. Significance was determined for three sample comparison of non-demented control and two MAPT IVS10+16 lines using one-way ANOVA followed by Tukey's test; * $=\mathrm{P}<0.05$; pair-wise comparison of the MAPT P301L line and its 
isogenic control was carried out using Student's $t$ test; ${ }^{*}=\mathrm{P}<0.05$; error bar represents s.e.m.; $n=3$ independent experiments.

(C) Acute depolymerisation of microtubules reverses nuclear lamina invaginations and restores rounded nuclear shapes. Confocal images of control and FTD-MAPT neurons (using genotypes described in A; 120 DIV), treated with DMSO (vehicle) or $10 \mu \mathrm{M}$ nocodazole for 3 hours (laminB1, green; $\beta 3$-tubulin, red; DAPI, blue). Scale bars $=10$ $\mu \mathrm{m}$.

(D) The proportion of FTD-MAPT neurons with nuclear lamina invaginations is significantly reduced by nocodazole treatment. Quantification of neurons with abnormalities of the nuclear lamina was carried out as in B. $n=3$ independent experiments; Error bars = s.e.m. Significance was determined using one-way ANOVA followed by Tukey's test; * $=\mathrm{P}<0.05$; error bar represents s.e.m.; $\mathrm{n}=3$ independent experiments).

See also Figure S3.

Figure 4. Super-resolution imaging demonstrates close apposition of tau and tubulin within nuclear lamina invaginations

(A) 3D-reconstructions using STED imaging of the nuclear lamina (laminB1; green) in FTD-MAPT iPSC-derived neurons (MAPT IVS10+16 and MAPT P301L) reveal pronounced nuclear invaginations compared with non-demented and MAPT P301isogenic control neurons (120 DIV). Scale bar $=10 \mu \mathrm{m}$.

(B) Tau is in close proximity to the nuclear lamina within nuclear invaginations of FTDMAPT neurons. STED imaging of control and FTD-MAPT iPSC-derived neurons (using 
genotypes described in A; 120 DIV; tau, green; $\beta 3$-tubulin, red; DNA, Yo-Pro, blue).

Detail from white boxes in upper panels, showing both merge of all channels, and single channel images of tau (green) or $\beta 3$-tubulin (red). Dashed lines indicate the boundary between the nucleus (Nu) and cytoplasm (Cy). Arrows indicate invaginations into the nucleus.

(C) Nuclear invaginations are lined with nuclear lamina and contain tau. STED imaging of MAPT IVS10+16-A neurons (120DIV; tau, green; and laminB1, red). Arrow indicates tau within a nuclear invagination, in close proximity to the laminB1-positive inner nuclear lamina.

Figure 5. Tau-containing nuclear lamina invaginations in neurons of the postmortem FTD-MAPT cerebral cortex

(A) Increased incidence of laminB1-positive nuclear invaginations in vivo, in postmortem FTD-MAPT IVS10+16 cortex compared to age-matched controls. Immunohistochemistry of laminB1 in (upper panels) frontal and (lower panels) temporal cortex from individuals with FTD due to the MAPT IVS10+16 mutation or age-matched controls (non-demented). Arrows indicate nuclei exhibiting nuclear invaginations.

(B) Percentage of invaginated nuclei in deep layers of frontal and temporal cortex of two control (green) and two MAPT IVS10+16 individuals (red), calculated from 20 individual imaging fields, Nuclei were scored by three observers, blinded to the identity of the post-mortem samples, and the averages of the three measurements are shown. Error represents s.e.m. 
(C) Hyperphosphorylated, AT8+ tau within nuclear lamina invaginations in neurons of the frontal cortex of individuals carrying MAPT IVS10+16 mutation. Representative neuron (upper panels), showing an extensive nuclear invagination (laiminB1, green;

DAPI, grey) containing hyperphosphorylated tau (AT8, red). Further examples of frontal cortex neurons with nuclear invaginations containing phosphorylated tau; laminB1 (green) and phosphorylated tau (AT8; red). Arrows indicate nuclear invaginations. Scale bars $=10 \mu \mathrm{m}$

See also Figure S4.

Figure 6. Disrupted nucleocytoplasmic transport in FTD-MAPT neurons

(A) Nuclear pores are clustered within nuclear membrane invaginations in FTD-MAPT neurons. Nuclear pore subunit NUP98 (green) remains co-localised with laminB1 (red) within nuclear invaginations in MAPT P301-isogenic neurons and MAPT P301L, (120 DIV). Arrows indicate nuclear membrane invaginations. Scale bars $=5 \mu \mathrm{m}$.

(B) Functional assay demonstrates disrupted nucleocytoplasmic transport in human iPSC-derived FTD-MAPT neurons. Schematic illustrates lentiviral vector for coexpression of NES:GFP (nuclear export signal fused to GFP) and NLS:RFP (nuclear localisation signal fused to RFP) within human neurons and relative distributions of both proteins in healthy neurons and in cells with defective nucleocytoplasmic transport. Representative confocal images of control and FTD-MAPT neurons, (MAPT IVS10+16A/B and MAPT P301L; all 120 DIV) expressing GFP:NES and RFP:NLS (GFP, green; RFP, red; $\beta 3$ tubulin). Greyscale images of NES-GFP and NLS-RFP localisation in representative cells of each genotype are shown: FTD-MAPT neurons show an 
increase of GFP within the nucleus, and a reduction in nuclear localisation of NLS-RFP.

Scale bars $=20 \mu \mathrm{m}$.

(C) Quantification of the nuclear:cytoplasmic ratio for both NES:GFP and NLS:RFP

demonstrate altered nuclear transport in FTD-MAPT genotypes relative to controls:

NES:GFP is increased in the nucleus of FTD-MAPT neurons, whereas NLS:RFP is

decreased (Significance was determined for three sample comparison of non-demented control and two MAPT IVS10+16 lines using one-way ANOVA followed by Dunnett's test; ${ }^{*}=\mathrm{P}<0.05 ;{ }^{* *}=\mathrm{P}<0.01 ;{ }^{* *}=\mathrm{P}<0.001$. Pair-wise comparison of the MAPT P301L line and its isogenic control was carried out using Student's $t$ test; ${ }^{*}=\mathrm{P}<0.05$; error bar represents s.e.m.; $\mathrm{n}=4$ independent experiments).

See also Figure S5. 
bioRxiv preprint doi: https://doi.org/10.1101/328187; this version posted May 22, 2018. The copyright holder for this preprint (which was not aCC-BY 4.0 International license.

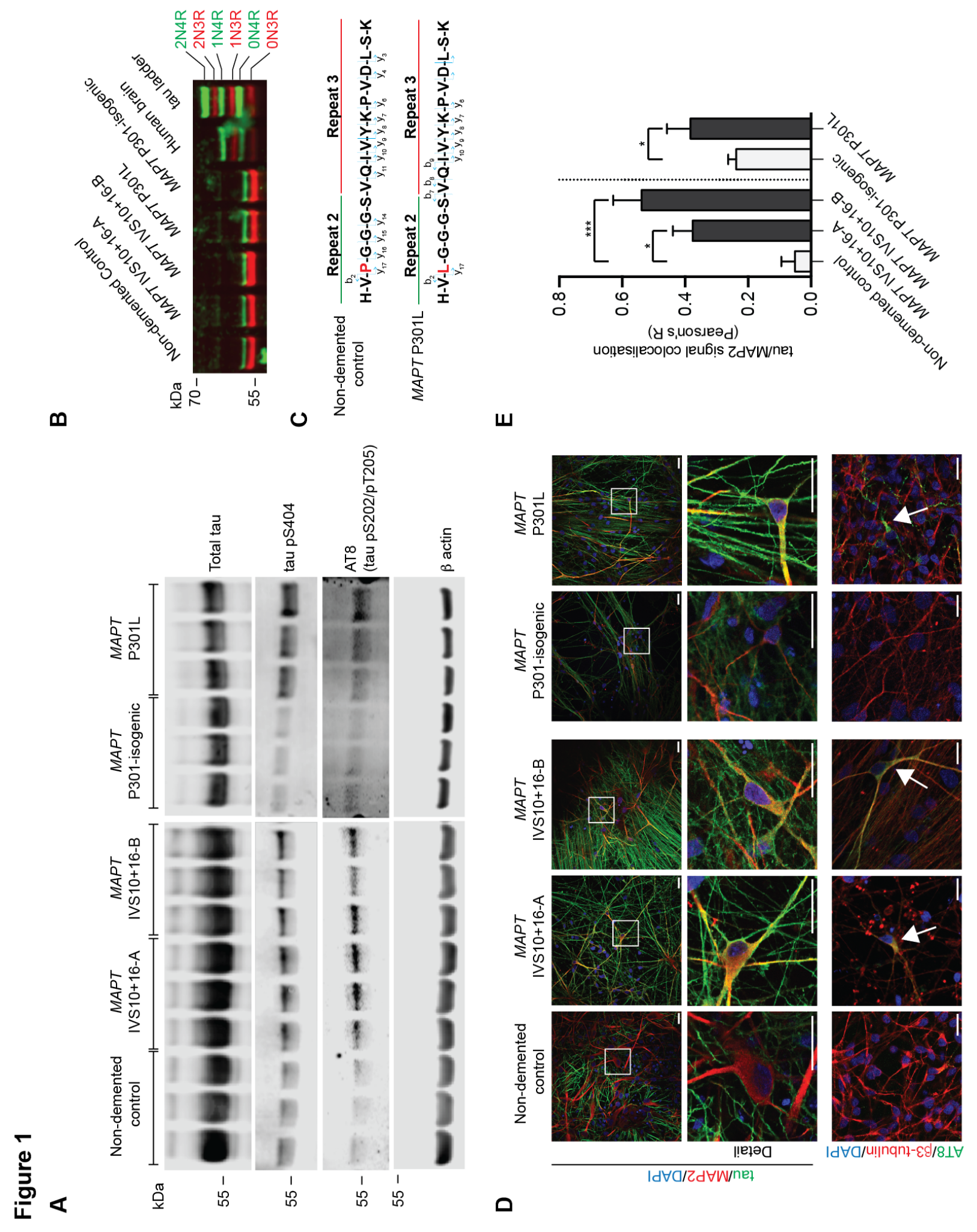



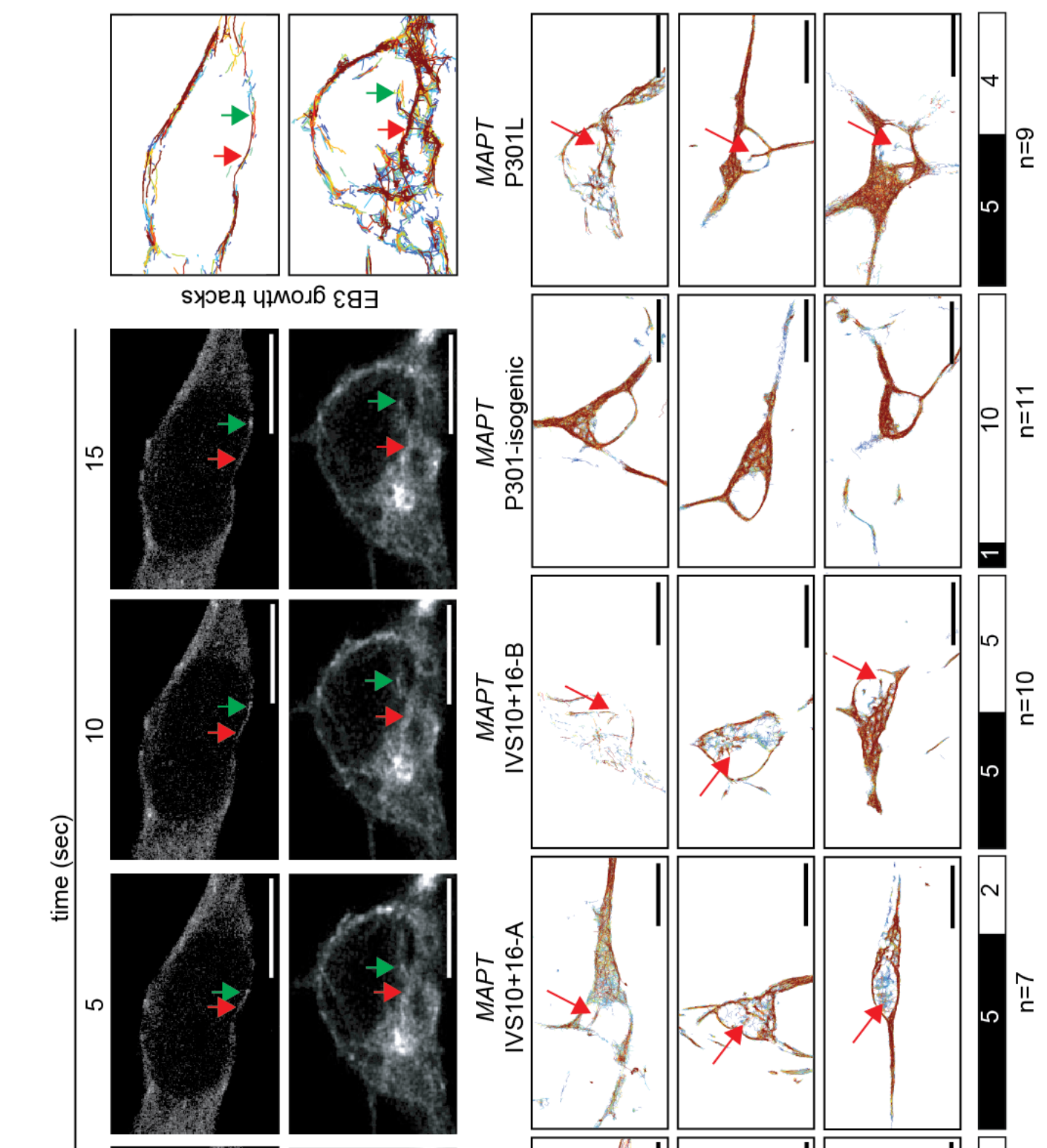

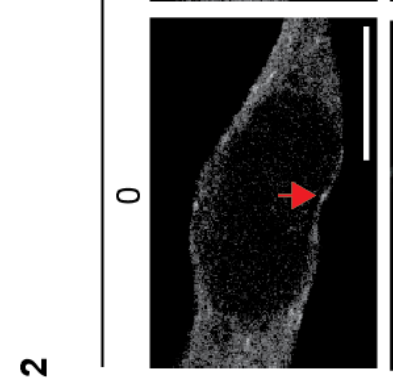

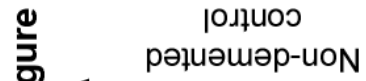

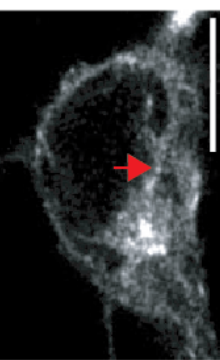

ᄀᄂo\&d

$\perp d \forall W$

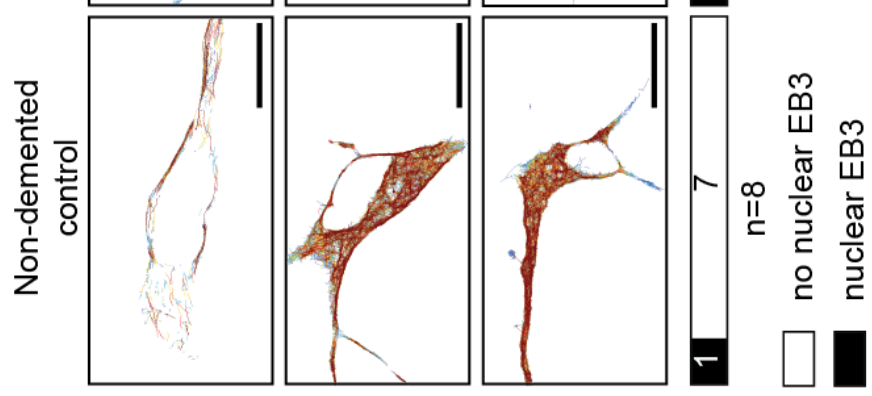

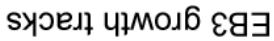

$\mathbf{m}$ 


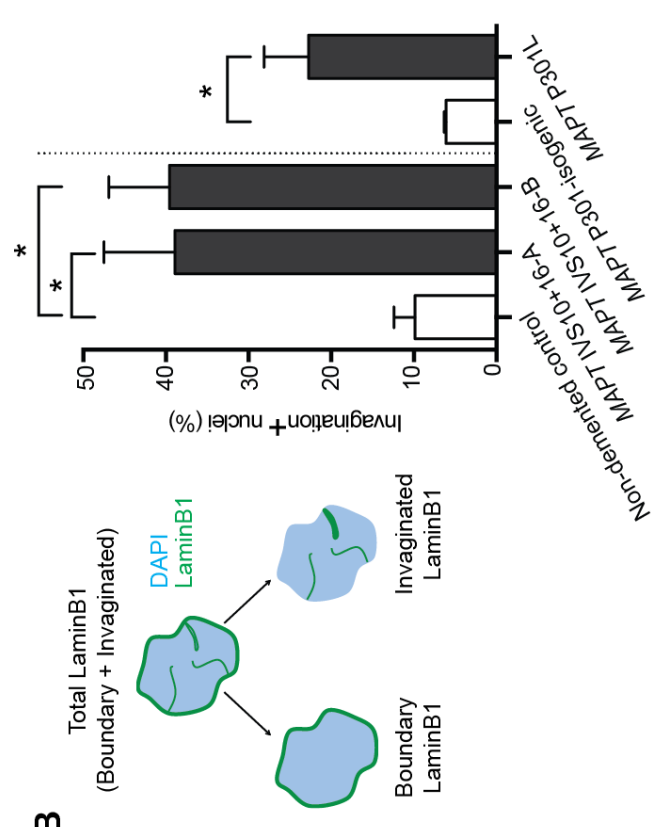

m
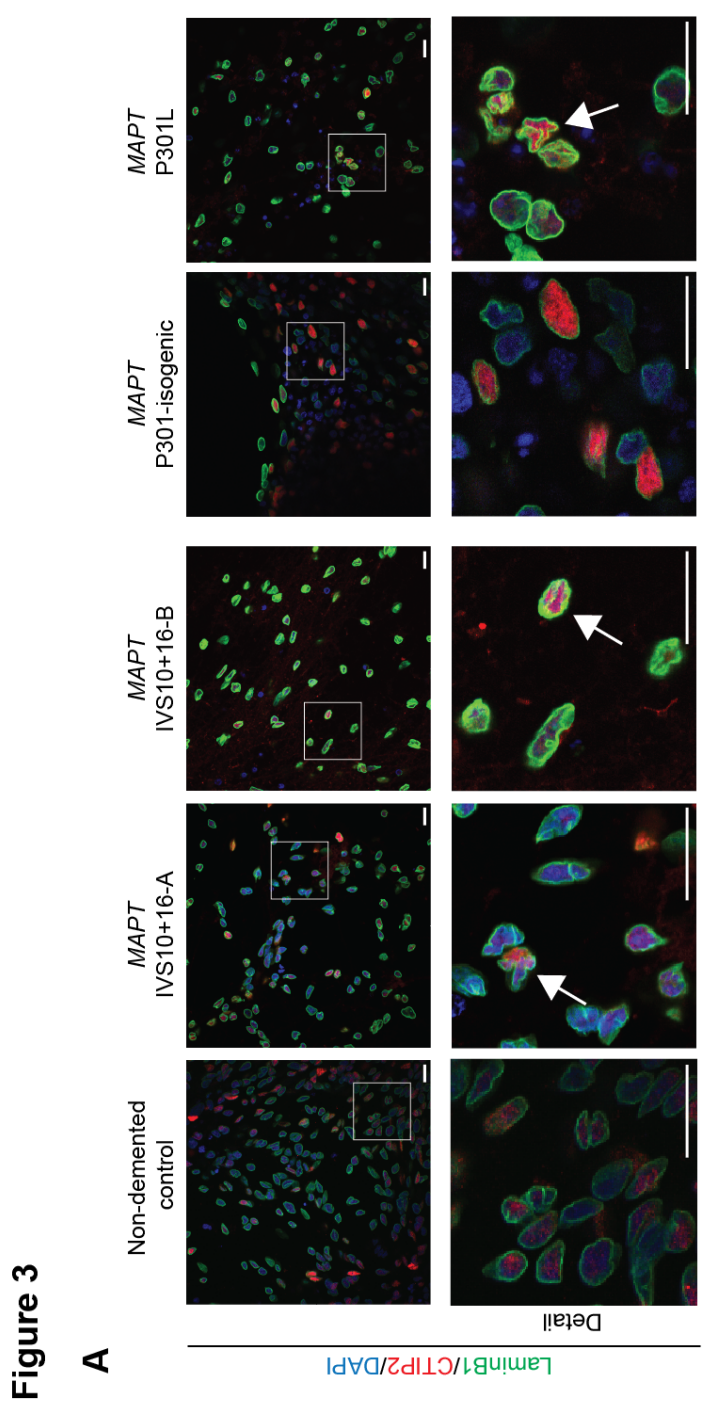

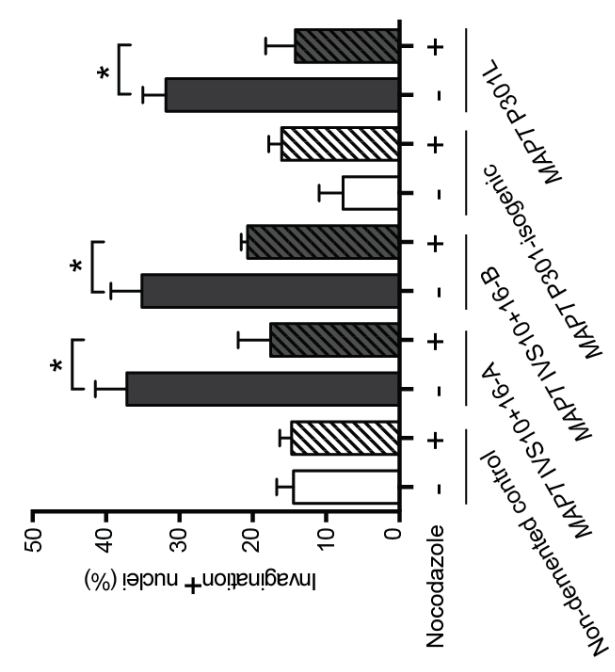

口
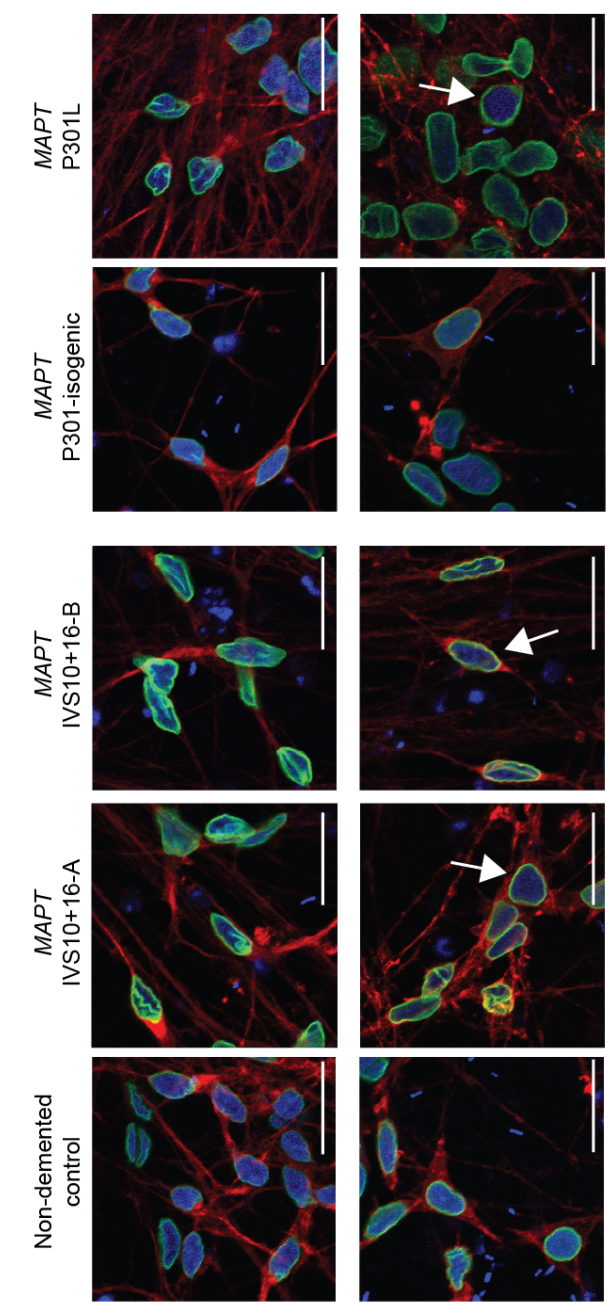

OSWO

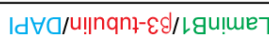



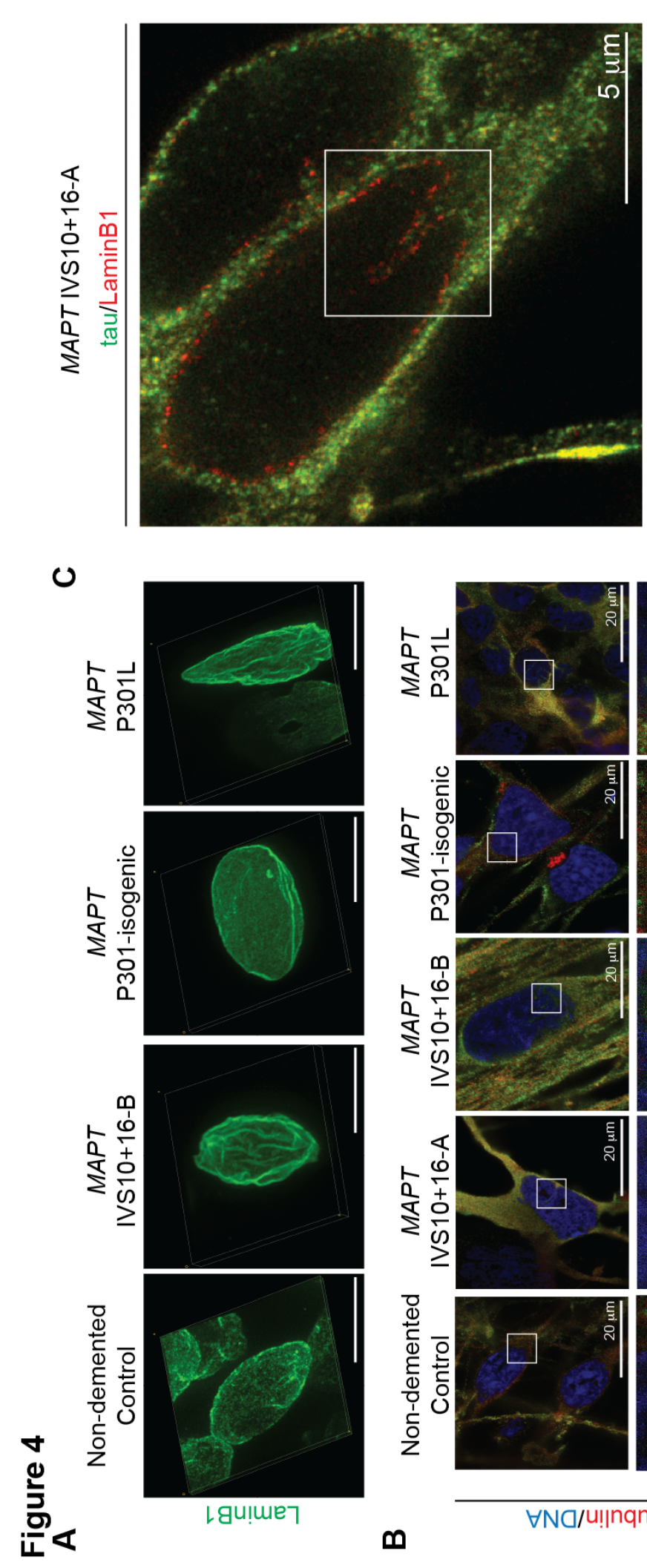

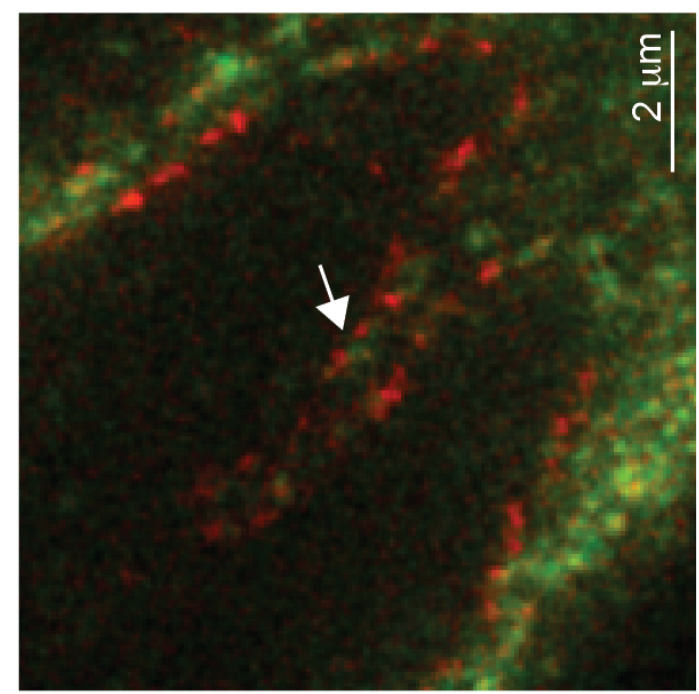

I!!ełə马

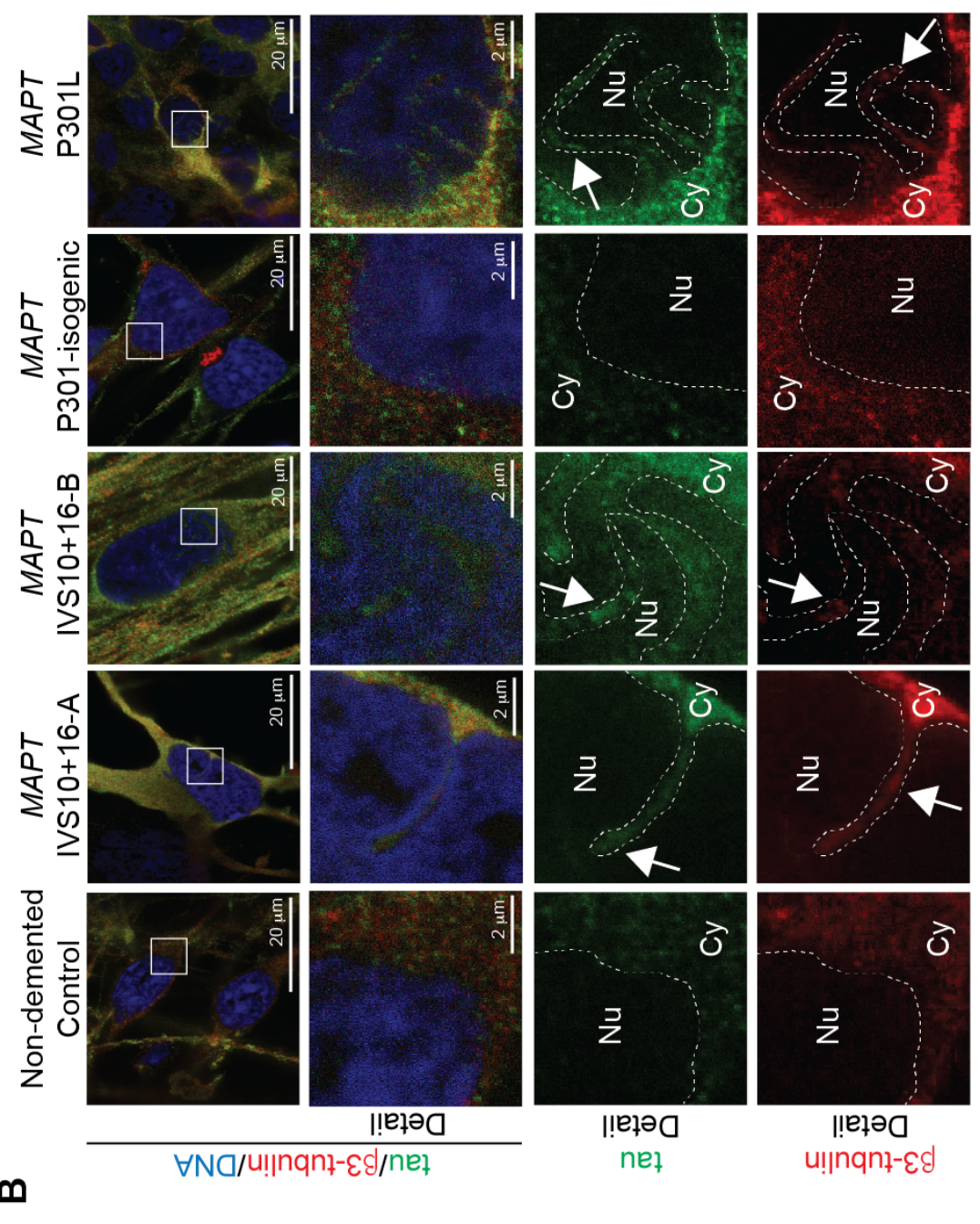




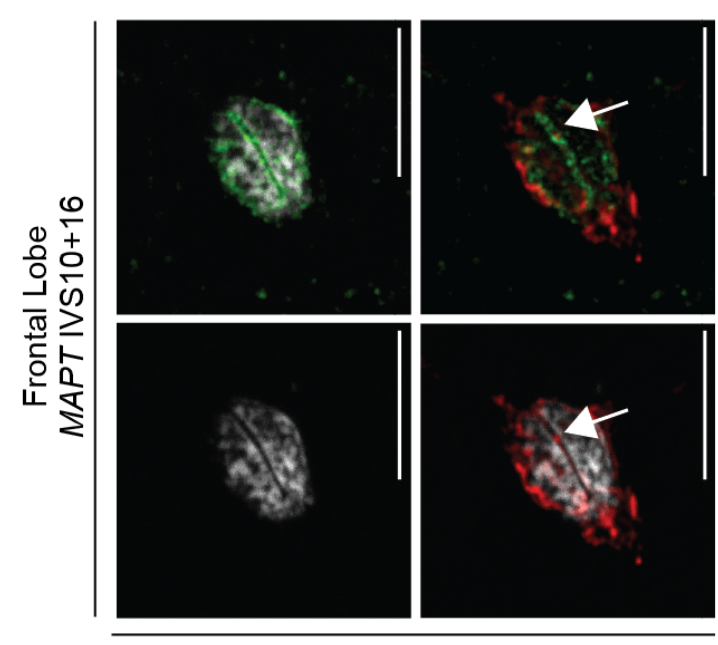

เgu!me $า / 8 \perp \forall /$ d $\forall 0$

0

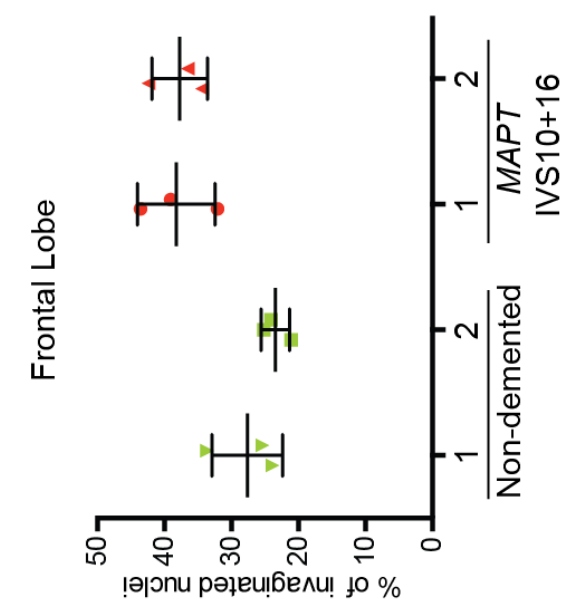

m

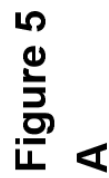

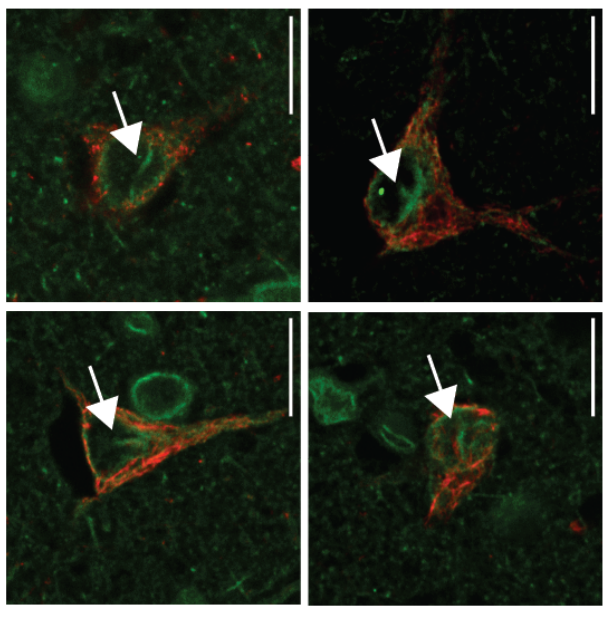

เgu!me $\urcorner / 8 \perp \forall$
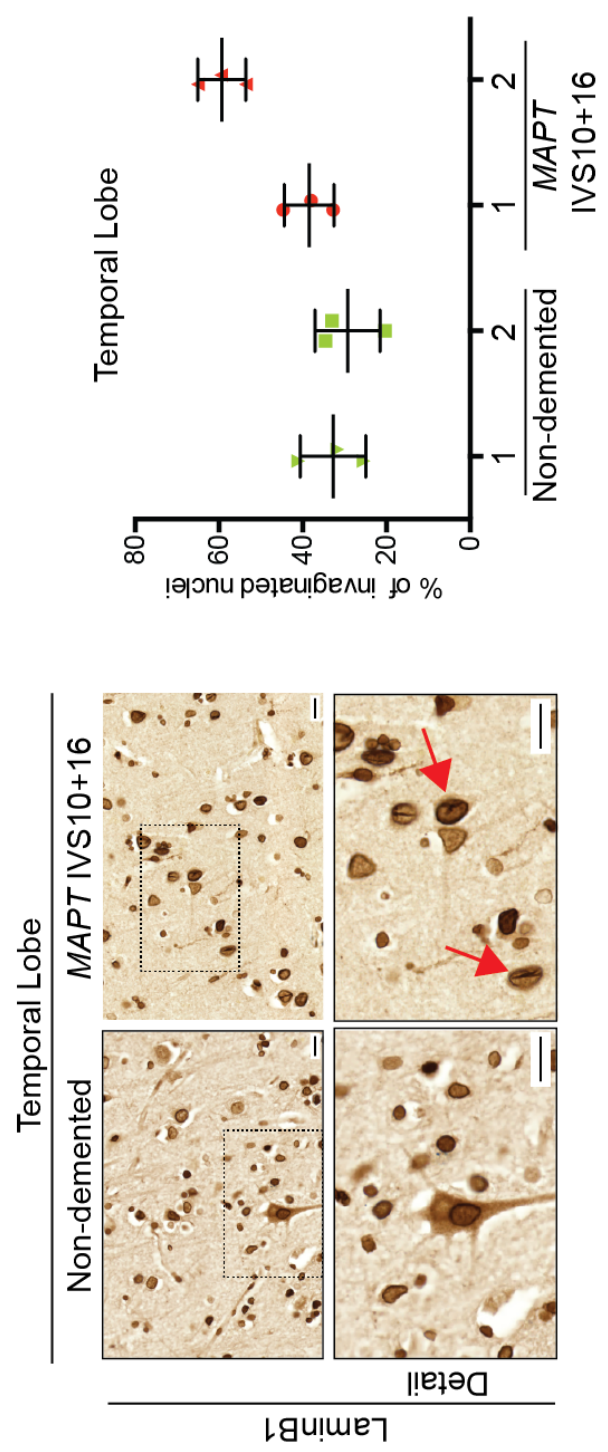
bioRxiv preprint doi: https://doi.org/10.1101/328187; this version posted May 22, 2018. The copyright holder for this preprint (which was not certified by peer review) is the author/funder, who has granted bioRxiv a license to display the preprint in perpetuity. It is made available under aCC-BY 4.0 International license.

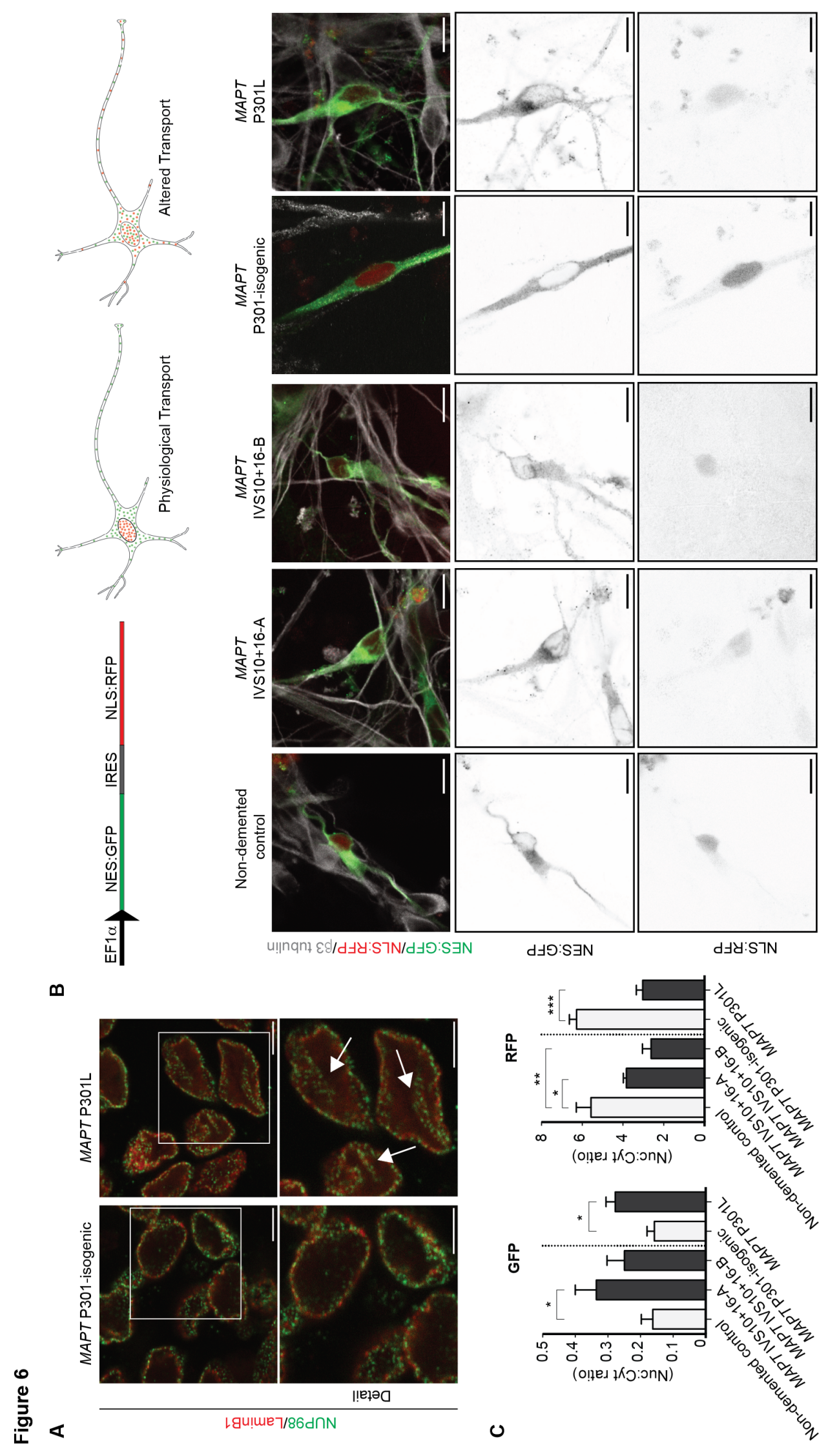

